\title{
ABELIAN UNIPOTENT SUBGROUPS OF FINITE UNITARY AND SYMPLECTIC GROUPS
}

\author{
W. J. WONG \\ (Received 25 September 1981) \\ Communicated by D. E. Taylor
}

\begin{abstract}
If $G$ is the unitary group $U(V)$ or the symplectic group $\operatorname{Sp}(V)$ of a vector space $V$ over a finite field of characteristic $p$, and $r$ is a positive integer, we determine the abelian $p$-subgroups of largest order in $G$ whose fixed subspaces in $V$ have dimension at least $r$, with the restriction that we assume $p \neq 2$ in the symplectic case. In particular, we determine the abelian subgroups of largest order in a Sylow $p$-subgroup of $G$. Our results complement earlier work on general linear and orthogonal groups.
\end{abstract}

1980 Mathematics subject classification (Amer. Math. Soc.): $20 \mathrm{G} 40$.

\section{Introduction}

Let $G$ be a classical group of linear transformations on a vector space $V$ over a finite field of characteristic $p$. For a fixed number $r$, consider the abelian $p$-subgroups of $G$ of largest order fixing the vectors of an $r$-dimensional subspace of $V$. These subgroups were determined in the cases when $G$ is a general linear group or an orthogonal group, by Goozeff (1970) and Wong (1981). In this paper we deal with the cases when $G$ is a unitary group or a symplectic group, where we assume $p \neq 2$ in the symplectic case.

Our methods are inductive, and are similar to those used in the orthogonal case, with some simplifications due to the fact that the underlying sesquilinear form is not symmetric. After some notation, terminology and preliminary remarks in Section 1, we study the unitary case, giving the orders of the relevant abelian p-subgroups in Section 2, describing the subgroups themselves in Section 3, and

c. Copyright Australian Mathematical Society 1982 
finding the largest abelian subgroups of a fixed Sylow $p$-subgroup of $G$ in Section 4. The corresponding results for the symplectic case are given in Section 5.

The case of the symplectic group in characteristic 2 has been studied independently by Barry and the present author, and is dealt with in the companion paper, Barry and Wong (1982), which follows this article.

\section{Preliminaries}

We take a finite field $F$ of characteristic $p$, with an automorphism $J$ such that $J^{2}=1$. Consider a finite-dimensional vector space $V$ over $F$ with an anti-Hermitian form $H: V \times V \rightarrow F$. Thus

$$
\begin{gathered}
H\left(v+v^{\prime}, w\right)=H(v, w)+H\left(v^{\prime}, w\right), \\
H(a v, w)=a H(v, w), \\
H(w, v)=-H(v, w)^{J},
\end{gathered}
$$

where $v, v^{\prime}, w \in V, a \in F$. We use Dieudonné (1955) as a basic reference. If $W, Z$ are subspaces of $V, W \subseteq Z$, we have the following glossary.

$W^{\perp}=$ orthogonal complement of $W$ in $V$, relative to $H$.

$W_{Z}^{\perp}=W^{\perp} \cap Z$.

Radical of $W, \operatorname{rad} W=W_{W}^{\perp}$.

$W$ is degenerate if $\operatorname{rad} W \neq 0$.

$W$ is totally isotropic if $\operatorname{rad} W=W$.

Isotropic vector: $v \neq 0, H(v, v)=0$.

The case $J=1$ occurs when $H$ is an alternating form, that is, $H(v, v)=0$ for all $v$. In this case, if $V$ is nondegenerate, then $V$ has even dimension $2 m$, and all maximal totally isotropic subspaces have dimension $m$. The isometry group, consisting of all linear transformations $\sigma$ on $V$ such that $H(\sigma v, \sigma w)=H(v, w)$ for all $v, w$ in $V$, is the symplectic group $\operatorname{Sp}(V)$.

If $J \neq 1$ and $V$ is nondegenerate, then $H$ is uniquely determined to within equivalence by the dimension of $V$, Dieudonne (1955), page 16, and all maximal totally isotropic subspaces have dimension $\left[\frac{1}{2} \operatorname{dim} V\right]$. The isometry group in this case is the unitary group $U(V)$.

We shall assume in the rest of this section and in the next three sections that we have the unitary case that $J \neq 1$ and $V$ is nondegenerate of dimension $n$. The symplectic case will be left to Section 5 .

Let $F_{0}$ be the fixed field of $J$, and set $q=\left|F_{0}\right|$, so that $|F|=q^{2}$, and $a^{J}=a^{q}$, $a \in F$. An element $a$ of $F$ is said to be skew if $a^{J}=-a$. Nonzero skew elements exist, since every element of the form $b^{J}-b$ is skew, and multiplication by such 
an element would convert $H$ into an Hermitian form, which is perhaps more commonly taken when the unitary group is defined.

LEMMA 1. Every skew element of $F$ has the form $b^{J}-b$, for exactly q elements $b$ of $F$.

Proof. The map $b \rightarrow b^{J}-b$ is an endomorphism $\psi$ of the additive group of $F$, with kernel $F_{0}$ of order $q$. Hence its image consists of $q$ elements. If $a$ is a nonzero skew element, the set of skew elements is $a F_{0}$. Thus the skew elements are precisely the elements of the image of $\psi$. This proves Lemma 1.

Sometimes we have to consider $V$ as a vector space over $F_{0}$, and then we speak of $F_{0}$-subspaces, $F_{0}$-dimension, and so on. The words subspace, dimension, $\ldots$, without prefix, will mean subspace over $F$, dimension over $F$, and so on. Also, we define, for $x, y \in V$,

$$
H_{0}(x, y)=H(x, y)-H(y, x)=\operatorname{tr} H(x, y),
$$

the trace of $H(x, y)$ in $F_{0}$. Then $H_{0}$ is an alternating $F_{0}$-bilinear form on $V$. We speak of $H_{0}$-orthogonality, $H_{0}$-totally isotropic subspaces, $H_{0}$-radical, and so on, to distinguish from the corresponding concepts relative to $H$, which we continue to write without prefixes.

Lemma 2. If $W$ is a subspace of $V$, then the $H_{0}$-radical of $W$ is the same as the radical of $W$. If $\operatorname{dim} W=s, \operatorname{dim}(\operatorname{rad} W)=h$, then the maximal $H_{0}$-totally isotropic $F_{0}$-subspaces of $W$ have $F_{0}$-dimension equal to $s+h$.

Proof. Let $x$ be an element of the $H_{0}$-radical of $W$. If $y \in W$, then $\operatorname{tr}(a H(x, y))=H_{0}(x, a y)=0$, for all $a \in F$. Since the trace map is nonzero, $H(x, y)=0$, and so $x$ lies in the radical of $W$. Since the radical is clearly contained in the $H_{0}$-radical, we have the first assertion. Now choose a subspace $Y$ such that

$$
W=(\operatorname{rad} W) \oplus Y,
$$

so that $\operatorname{dim}_{F_{0}} Y=2 \operatorname{dim} Y=2(s-h)$. The maximal $H_{0}$-totally isotropic $F_{0}$-subspaces $T$ of $W$ have the form

$$
T=(\operatorname{rad} W) \oplus U,
$$

where $U$ is a maximal $H_{0}$-totally isotropic $F_{0}$-subspace of $Y$. Since $Y$ is nondegenerate relative to $H_{0}$,

$$
\operatorname{dim}_{F_{0}} U=\frac{1}{2} \operatorname{dim}_{F_{0}} Y=s-h .
$$

Since $\operatorname{dim}_{F_{0}}(\operatorname{rad} W)=2 h$, we have $\operatorname{dim}_{F_{0}} T=s+h$. This proves Lemma 2 . 


\section{Orders}

As in Wong (1981), we say an element or subgroup of the unitary group $U(V)$ fixes a subspace $X$ of $V$ if it fixes every vector in $X$. More generally, it fixes a quotient $X / Y$ of subspaces if it maps every coset $x+Y$ on itself, $x \in X$. For $0 \leqslant r \leqslant n$, we consider the abelian $p$-subgroups $A$ of $U(V)$ such that $A$ fixes some subspace of dimension $r$, and let $\mathcal{Q}(V, r)$ be the set of those $A$ having the largest possible order. We denote this order as $q^{f(n, r)}$, where $q$ is the number of elements in the fixed field $F_{0}$ of $J$.

The following result is proved as in Wong (1981).

Lemma 3. If $A$ is a p-subgroup of $U(V), A \neq 1$, then the vectors of $V$ fixed by $A$ form a degenerate subspace. In particular, A fixes some isotropic vector.

If $x$ is an isotropic vector, we can choose another isotropic vector $y$, such that $H(x, y)=1$, by Dieudonné (1955), page 20. Then $x, y$ is called a hyperbolic pair, and spans a nondegenerate subspace $\langle x, y\rangle$. If $t \in V$, then $H(t, t)$ is a skew element of $F$ (since $H$ is anti-Hermitian), and so

$$
B(t)=\left\{b \in F \mid b^{J}-b=H(t, t)\right\}
$$

is a subset of $F$ having $q$ elements, by Lemma 1 .

Lemma 4. Suppose $x, y$ is a hyperbolic pair in $V$, and $Z=\langle x, y\rangle^{\perp}$, so that $V=\langle x\rangle \oplus Z \oplus\langle y\rangle$. For $\mu \in U(Z), t \in Z, b \in B(t)$, define a linear transformation $\sigma(\mu, t, b)$ on $V$ by

$$
\begin{aligned}
& \sigma(\mu, t, b) x=x \\
& \sigma(\mu, t, b) z=\mu z-H(\mu z, t) x, \quad z \in Z, \\
& \sigma(\mu, t, b) y=y+t+b x
\end{aligned}
$$

Then (i) $\sigma(\mu, t, b)$ is a unitary transformation fixing $x$, and, conversely, every unitary transformation of $V$ fixing $x$ has the form $\sigma(\mu, t, b)$, for unique $\mu \in U(Z)$, $t \in Z, b \in B(t)$.

(ii) $\sigma(\mu, t, b) \sigma\left(\mu^{\prime}, t^{\prime}, b^{\prime}\right)=\sigma\left(\mu \mu^{\prime}, t+\mu t^{\prime}, b+b^{\prime}-H\left(\mu t^{\prime}, t\right)\right)$.

(iii) $\sigma(\mu, t, b)$ and $\sigma\left(\mu^{\prime}, t^{\prime}, b^{\prime}\right)$ commute if and only if $\mu \mu^{\prime}=\mu^{\prime} \mu,(\mu-1) t^{\prime}=$ $\left(\mu^{\prime}-1\right) t$, and $H\left(\mu^{\prime} t, t^{\prime}\right)=H\left(\mu t^{\prime}, t\right)$.

(iv) If $W$ is a subspace of $Z$ and $X=\langle x\rangle \oplus W$, then $\sigma(\mu, t, b)$ fixes $X$ if and only if $\mu$ fixes $W$ and $t \in W_{Z}^{\perp}$. 
Proof. Any unitary transformation $\sigma$ fixing $x$ must leave $\langle x\rangle^{\perp}=\langle x\rangle \oplus Z$ invariant, and so must have the form

$$
\begin{aligned}
\sigma x & =x, \\
\sigma z & =\mu z+f(z) x, \quad z \in Z, \\
\sigma y & =a y+t+b x,
\end{aligned}
$$

where $\mu: Z \rightarrow Z, f: Z \rightarrow F$ are linear maps, $t \in Z$, and $a, b \in F$. Easy calculation shows that $\sigma \in U(V)$ if and only if $\mu \in U(Z), f(z)=-H(\mu z, t), a=1$ and $b \in B(t)$, so that $\sigma=\sigma(\mu, t, b)$. The multiplication formula is a straightforward computation, and the last two assertions are obvious. This proves Lemma 4.

Lemma 5. In the situation of Lemma 4, let $A$ be an abelian subgroup of $U(V)$ fixing $x$. Let $T=\{t \in Z \mid \sigma(1, t, b) \in A$, some $b\}$, let $S$ be the subspace of $Z$ spanned by $T$, and let $S_{0}$ be the $F_{0}$-subspace of $Z$ spanned by $T$. Set $s=\operatorname{dim}_{F} S$, $s_{0}=\operatorname{dim}_{F_{0}} S_{0}, h=\operatorname{dim}_{F}(\operatorname{rad} S)$. Then

(i) If $\sigma(\mu, t, b) \in A$, then $\mu$ fixes $S$, and $t$ is $H_{0}$-orthogonal to $S_{0}$.

(ii) $S_{0}$ is $H_{0}$-totally isotropic.

(iii) $h \leqslant \min (s, n-2-s), s_{0} \leqslant s+h$.

Proof. If $\sigma(\mu, t, b), \sigma\left(1, t^{\prime}, b^{\prime}\right) \in A$, then, since $A$ is abelian, $(\mu-1) t^{\prime}=0$, by Lemma 4(iii). Hence $\mu$ fixes $T$, and so $\mu$ fixes $S$. Also, $H\left(t^{\prime}, t\right)=H\left(\mu t^{\prime}, t\right)=$ $H\left(t, t^{\prime}\right)$, so that $H_{0}\left(t, t^{\prime}\right)=0$. Hence $t$ is $H_{0}$-orthogonal to $S_{0}$. In particular, $T$ is $H_{0}$-orthogonal to $S_{0}$, and so $S_{0}$ is $H_{0}$-totally isotropic. This proves (i) and (ii).

Since $\operatorname{rad} S \subseteq S$ and $\operatorname{rad} S \subseteq S_{Z}^{1}$, we have $h \leqslant s$ and $h \leqslant n-2-s$. By Lemma $2, s_{0} \leqslant s+h$. This proves Lemma 5 .

We now find an upper bound for $f(n, r)$, where we may assume $n>r \geqslant 1$, by Lemma 3.

LEMMA 6. Let $n>r \geqslant 1$, and set

$$
g(n, r, s)=1+\min (2 s, n-2)+f(n-2, \max (r-1, s)) .
$$

Then $f(n, r) \leqslant \max \{g(n, r, s) \mid 0 \leqslant s \leqslant n-r-1\}$.

Proof. We take $A$ in $\mathscr{Q}(V, r)$, and may assume $A \neq 1$. By Lemma 3 , we choose a degenerate $r$-dimensional subspace $X$ fixed by $A$, and take a nonzero vector $x$ in $\operatorname{rad} X$. Take $y$ and $Z$ as in Lemma 4 , and define the transformations $\sigma(\mu, t, b)$. Set $W=X \cap Z$, so that $X=\langle x\rangle \oplus W$.

The map $\sigma(\mu, t, b) \rightarrow \mu$ is a homomorphism of $A$ onto an abelian $p$-subgroup $A_{1}$ of $U(Z)$, whose kernel $M$ consists of all $\sigma(1, t, b)$ which lie in $A$. Let $T, S, S_{0}$, 
$s, s_{0}, h$ be defined as in Lemma 5 . By Lemmas 4 and 5, $A_{1}$ fixes both $W$ and $S$, so that

$$
\left|A_{1}\right| \leqslant q^{f(n-2, d)}
$$

where $d=\max (r-1, s)$. Also, $M \subseteq\left\{\sigma(1, t, b) \mid t \in S_{0}, b \in B(t)\right\}$, so that

$$
|M| \leqslant q^{1+s_{0}} \leqslant q^{1+s+h} \leqslant q^{1+e},
$$

where $e=\min (2 s, n-2)$, by Lemma 5 . Hence,

$$
|A|=|M|\left|A_{1}\right| \leqslant q^{g(n, r, s)} .
$$

Since $S \subseteq W_{Z}^{\perp}$ by Lemma 4 , it follows that $0 \leqslant s \leqslant n-r-1$. This proves Lemma 6.

Let $\delta(V)$ be the set of all subspaces $X$ of $V$ such that $X \supseteq X^{\perp}$ or $X \subseteq X^{\perp}$, that is, $X$ is a totally isotropic subspace or the orthogonal complement of a totally isotropic subspace. Let $\mathcal{S}_{r}(V)$ be the set of all $r$-dimensional subspaces in $\delta(V)$. We have the following simple result.

LemMA 7. Let $r, s$ be nonnegative integers, $r+s \leqslant n, d=\max (r, s)$, and $X \in \delta_{d}(V)$. Then there exist subspaces $W, S$ of $X$, such that $W \in \mathcal{S}_{r}(V), S \in \mathcal{S}_{s}(V)$, and $W$ is orthogonal to $S$.

Proof. By symmetry, we may assume $r \geqslant s$. Then, since $X \in \delta(V)$, $\operatorname{dim}(\operatorname{rad} X)=\min (r, n-r) \geqslant s$. We take $W$ to be $X$, and $S$ to be any $s$-dimensional subspace of $\operatorname{rad} X$. This proves Lemma 7 .

LEMMA 8 (Recursion Formula). $f(n, 0)=f(n, 1), f(n, n)=0$. If $1 \leqslant r<n$, then

$$
f(n, r)=\max \{g(n, r, s) \mid 0 \leqslant s \leqslant n-r-1\},
$$

where $g(n, r, s)=1+\min (2 s, n-2)+f(n-2, \max (r-1, s))$. There exist $X \in \mathcal{S}_{r}(V)$ and $A \in \mathcal{Q}(V, r)$ such that $A$ fixes $X$.

Proof. Clearly $f(n, n)=0$, while $f(n, 0)=f(n, 1)$ by Lemma 3 , and the last assertion holds for $r=n, 0,1$. In particular, the result holds for $n \leqslant 1$. We suppose $n \geqslant 2$, and use induction. Suppose $1 \leqslant r<n$ and $0 \leqslant s \leqslant n-r-1$. We can choose an isotropic vector $x$ in $V$, by Dieudonné (1955), page 16, and choose $y, Z$ as in Lemma 4. Let $d=\max (r-1, s)$. By induction, there exist a subspace $Y \in \varsigma_{d}(Z)$ and an abelian $p$-subgroup $A_{1}$ of $U(Z)$ of order $q^{f(n-2, d)}$ fixing $Y$. By Lemma $7, Y$ has subspaces $W, S$ which are orthogonal to each other, such that 
$W \in \varsigma_{r-1}(Z), S \in \mathcal{S}_{s}(Z)$. Since $\operatorname{dim}(\operatorname{rad} S)=\min (s, n-2-s), S$ has an $H_{0^{-}}$ totally isotropic $F_{0}$-subspace $S_{0}$ of $F_{0}$-dimension $s_{0}=s+\min (s, n-2-s)=$ $\min (2 s, n-2)$. Then

$$
A=\left\{\sigma(\mu, t, b) \mid \mu \in A_{1}, t \in S_{0}, b \in B(t)\right\}
$$

is a set of $q^{g(n, r, s)}$ elements of $U(V)$, fixing the subspace $X=\langle x\rangle \oplus W$, which lies in $\mathcal{S}_{r}(V)$. Since $A_{1}$ fixes $S_{0}$, it is easy to check that $A$ is closed under multiplication, so that $A$ is a $p$-subgroup of $U(V)$. Since $A_{1}$ is abelian, $A_{1}$ fixes $S_{0}$, and $S_{0}$ is $H_{0}$-totally isotropic, Lemma 4 shows that $A$ is abelian. Hence, $f(n, r) \geqslant g(n, r, s)$. Application of Lemma 6 completes the proof of Lemma 8.

The equality in the Recursion Formula implies that the inequalities for $\left|A_{1}\right|$ and $|M|$ in the proof of Lemma 6 are in fact equalities. Thus

$$
\begin{gathered}
A_{1} \in \mathbb{Q}(Z, \max (r-1, s)), \\
M=\left\{\sigma(1, t, b) \mid t \in S_{0}, b \in B(t)\right\}, \\
s_{0}=s+h, \quad h=\min (s, n-2-s) .
\end{gathered}
$$

Hence $S \in \delta_{s}(Z)$, and $S_{0}$ is a maximal $H_{0}$-totally isotropic $F_{0}$-subspace of $S$.

THEOREM 1. Let $U(V)$ be a finite unitary group, where $V$ is an n-dimensional vector space over a field of $q^{2}$ elements having characteristic $p$, and let $q^{f(n, r)}$ be the largest order of an abelian p-subgroup of $U(V)$ fixing an r-dimensional subspace of $V$.

(a) If $r \geqslant \frac{1}{2} n$, then $f(n, r)=(n-r)^{2}$.

(b) If $n$ is even and $r<\frac{1}{2} n$, then $f(n, r)=\frac{1}{4} n^{2}$.

(c) If $n$ is odd, $n \geqslant 3$ and $r<\frac{1}{2} n$, then $f(n, r)=\frac{1}{4}(n-1)^{2}+1$; and $f(1,0)=0$.

Proof. The result holds for $n \leqslant 1$ or $r=n$. We may suppose $n \geqslant 2,1 \leqslant r<n$, and use induction on $n$.

First suppose $r \geqslant \frac{1}{2} n$. If $0 \leqslant s \leqslant n-r-1$, then $s \leqslant r-1$ and $2 s \leqslant n-2$. Thus, $g(n, r, s)=1+2 s+f(n-2, r-1)$. In the expression for $f(n, r)$ given in the Recursion Formula, the maximum value of $g(n, r, s)$ occurs at $s=n-r-1$, and $f(n, r)=2 n-2 r-1+f(n-2, r-1)$. By inductive hypothesis,

$$
f(n-2, r-1)=(n-r-1)^{2} .
$$

Hence, $f(n, r)=(n-r)^{2}$.

Now let $r<\frac{1}{2} n$. If $s<\frac{1}{2}(n-2)$, then $f(n-2, \max (r-1, s))$ is a constant function of $s$, by inductive hypothesis, and so

$$
g(n, r, s)=1+2 s+f(n-2, \max (r-1, s))
$$


is a strictly increasing function of $s$ in this range. If $\frac{1}{2}(n-2) \leqslant s \leqslant n-r-1$, then $s>r-1$, and so

$$
g(n, r, s)=1+n-2+f(n-2, s)=n-1+(n-2-s)^{2},
$$

a strictly decreasing function of $s$ in this range. Thus the maximum value of $g(n, r, s)$ occurs at most at the values $s=\left[\frac{1}{2}(n-3)\right],\left[\frac{1}{2}(n-1)\right]$. A check of values shows that, if $n$ is even, then $g\left(n, r, \frac{1}{2}(n-4)\right)=\frac{1}{4} n^{2}-2, g\left(n, r, \frac{1}{2}(n-2)\right)$ $=\frac{1}{4} n^{2}$, so that the maximum occurs only at $s=\frac{1}{2}(n-2)$, and $f(n, r)=\frac{1}{4} n^{2}$. If $n$ is odd, then $g\left(n, r, \frac{1}{2}(n-3)\right)=g\left(n, r, \frac{1}{2}(n-1)\right)=\frac{1}{4}(n-1)^{2}+1$, so the maximum occurs at $s=\frac{1}{2}(n-3), \frac{1}{2}(n-1)$, and $f(n, r)=\frac{1}{4}(n-1)^{2}+1$. (If $n=3$, only the case $s=\frac{1}{2}(n-1)$ occurs. $)$ This proves Theorem 1 .

\section{Groups}

We let $A(X)$ denote the subgroup of all elements of $U(V)$ fixing a subspace $X$ of $V$. More generally, if $Z$ is a nondegenerate subspace of $V$ containing $X$, we let $A_{Z}(X)$ denote the subgroup of $U(Z)$ fixing $X$. Also, if $X$ is a totally isotropic subspace of $V$ and $\mathcal{E}$ is an $F_{0}$-subspace of the space of all linear transformations of $X^{\perp} / X$ into $X$, we write $A\left(X, X^{\perp} / X ; \mathcal{L}\right)$ for the set of all elements $\sigma$ of $U(V)$ which fix both $X$ and $X^{\perp} / X$, and for which the map of $X^{\perp} / X$ into $X$ induced by

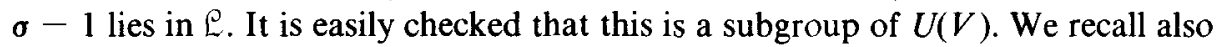
that $\mathcal{S}_{r}(V)$ denotes the set of all $r$-dimensional subspaces of $V$ which are either totally isotropic or orthogonal complements of totally isotropic subspaces.

THEOREM 2. Let $U(V)$ be the unitary group of an n-dimensional vector space $V$ over a finite field of characteristic $p$, and let $\mathcal{Q}(V, r)$ be the set of all abelian p-subgroups of largest order in $U(V)$ fixing an $r$-dimensional subspace of $V$.

(a) If $r \geqslant \frac{1}{2} n$, then $\mathcal{Q}(V, r)=\left\{A(X) \mid X \in \mathcal{S}_{r}(V)\right\}$.

(b) If $n$ is even and $r<\frac{1}{2} n$, then $\mathbb{Q}(V, r)=\mathbb{Q}\left(V, \frac{1}{2} n\right)$.

(c) If $n$ is odd, $n \geqslant 3$, and $r<\frac{1}{2} n$, then $\mathbb{Q}(V, r)$ consists of all $A\left(X, X^{\perp} / X\right.$; $)$, where $X$ is a totally isotropic subspace of dimension $\frac{1}{2}(n-1)$ in $V$, and $\mathcal{E}$ is an $F_{0}$-subspace of $F_{0}$-dimension 1 in the space of all linear transformations of $X^{\perp} / X$ into $X$.

Proof. The result is true for $n \leqslant 1$ or $r=n$. We may assume $n \geqslant 2$ and $1 \leqslant r<n$, and use induction on $n$.

First suppose $r \geqslant \frac{1}{2} n$. If $X \in \mathcal{S}_{r}(V)$, so that $X \supseteq X^{\perp}$, we choose a nonzero vector $x$ in $X^{\perp}$, and take $y, Z$ as in Lemma 4. If $W=X \cap Z$, then $W \in \mathcal{S}_{r-1}(Z)$, 
and

$$
A(X)=\left\{a(\mu, t, b) \mid \mu \in A_{Z}(W), t \in W_{Z}^{\perp}, b \in B(t)\right\} .
$$

By inductive hypothesis, $A_{Z}(W) \in \mathscr{Q}(Z, r-1)$. As in the proof of Lemma 8 , $A(X)$ is an abelian $p$-subgroup of $U(V)$, and a calculation of its order $|A(X)|=$ $\left|A_{Z}(W)\right|\left|W_{Z}^{\perp}\right| q$ shows that $A(X) \in \mathbb{Q}(V, r)$.

Conversely, suppose $A \in \mathbb{Q}(V, r)$, take a degenerate $r$-dimensional subspace $X$ fixed by $A$, choose a nonzero vector $x$ in $\operatorname{rad} X$, and set up the situation of the proof of Lemma 6 . By the proof of Theorem $1, s=n-r-1 \leqslant \frac{1}{2}(n-2)$. By the remark following the proof of Lemma $8, S \in \mathfrak{S}_{s}(Z)$. Thus, $S$ is totally isotropic. Since $W \subseteq S_{Z}^{\perp}$ and $\operatorname{dim} W=r-1$, we have $W=S_{Z}^{\perp}$, so that $W \supseteq W_{Z}^{\perp}$. Hence $X \supseteq X^{\perp}$, so that $X \in \mathcal{S}_{r}(V)$. Since $A \subseteq A(X)$ and $A(X) \in \mathbb{Q}(V, r)$, we have $A=A(X)$. This completes the proof in case (a).

Now suppose $n$ is even and $r<\frac{1}{2} n$. By Theorem 1, $Q(V, r) \supseteq \mathcal{Q}\left(V, \frac{1}{2} n\right)$. Suppose $A \in Q(V, r)$, and set up the situation of Lemma 6 . By the proof of Theorem 1, $s=\frac{1}{2}(n-2)$. Since $S \in \mathcal{S}_{s}(Z), S$ is a maximal totally isotropic subspace of $Z$, and $S_{0}=S$ is a maximal $H_{0}$-totally isotropic $F_{0}$-subspace of $Z$. If $\sigma(\mu, t, b) \in A$, then $\mu$ fixes $S$, and $t$ is $H_{0}$-orthogonal to $S_{0}$, so that $t \in S_{0}=S$. Hence $A$ fixes $\langle x\rangle \oplus S$, which has dimension $\frac{1}{2} n$, so that $A \in Q\left(V, \frac{1}{2} n\right)$. This completes the proof in case (b).

Suppose $n$ is odd, $n \geqslant 3$, and $r<\frac{1}{2} n$. Let $X$ be a totally isotropic subspace of dimension $\frac{1}{2}(n-1)$ in $V$, and $E$ an $F_{0}$-subspace of $F_{0}$-dimension 1 in the space of all linear transformations of $X^{\perp} / X$ into $X$. Choose a nonzero element $\tau$ in $巳$ and a nonzero vector $x$ in $\tau\left(X^{\perp} / X\right)$. Take $y, Z$ as in Lemma 4, $W=X \cap Z$. If $z \in X^{\perp}$, denote its image in $X^{\perp} / X$ by $\bar{z}$. If $\sigma=\sigma(\mu, t, b)$ lies in $A\left(X, X^{\perp} / X\right.$; $\left.巳\right)$, then $t \in W_{Z}^{\perp}, \mu$ fixes $W_{Z}^{\perp}$ (since $\sigma$ fixes $X^{\perp} /\langle x\rangle$ ), and, for each $z \in W_{Z}^{\perp}$, there exists $c \in F_{0}$ such that $-H(z, t) x=c \tau(\bar{z})$. Conversely, if $\mu, t$ satisfy these conditions, then $\sigma(\mu, t, b)$ lies in $A\left(X, X^{\perp} / X ; \mathcal{E}\right)$ (since $W_{Z}^{\perp}$ covers $\left.X^{\perp} / X\right)$. Choose $w \in W_{Z}^{\perp}$ such that $\tau(\bar{w})=x$. Since $\bar{x}$ spans $X^{\perp} / X$, we see that

$$
\begin{aligned}
& A\left(X, X^{\perp} / X ; \mathcal{L}\right) \\
& \quad=\left\{\sigma(\mu, t, b) \mid \mu \in A_{Z}\left(W_{Z}^{\perp}\right), t \in W_{Z}^{\perp}, H(w, t) \in F_{0}, b \in B(t)\right\} .
\end{aligned}
$$

The map $t \rightarrow H(w, t)$ is an $F_{0}$-linear map of $W_{Z}^{\perp} \rightarrow F$ with kernel $W$. The inverse image of $F_{0}$ under this map is an $F_{0}$-subspace $S_{0}$ of $F_{0}$-dimension $n-2$ in $W_{Z}^{\perp}$. Since the totally isotropic space $W$ has $F_{0}$-codimension 1 in $S_{0}, S_{0}$ is $H_{0}$-totally isotropic. Now,

$$
A\left(X, X^{\perp} / X ; \varrho\right)=\left\{\sigma(\mu, t, b) \mid \mu \in A_{Z}\left(W_{Z}^{\perp}\right), t \in S_{0}, b \in B(t)\right\},
$$

where we know $A_{Z}\left(W_{Z}^{\perp}\right) \in \mathcal{Q}\left(Z, \frac{1}{2}(n-1)\right)$ by case (a). As in the proof of Lemma $8, A\left(X, X^{\perp} / X ; \mathcal{L}\right)$ is an abelian $p$-subgroup of $U(V)$, fixing $X$, and a calculation of its order shows that $A\left(X, X^{\perp} / X ; \mathcal{e}\right) \in \mathcal{Q}(V, r)$. 
We note incidentally that every element of $\mathcal{L}$ is induced by $\sigma-1$ for some $\sigma$ in $A\left(X, X^{\perp} / X ; \mathfrak{L}\right)$, since $t \rightarrow H(w, t)$ is a surjective map of $S_{0}$ on $F_{0}$.

Now suppose that $A \in \mathbb{Q}(V, r)$, and again set up the situation of Lemma 6 . First suppose $s=\frac{1}{2}(n-1)$. Since $S \in \mathcal{S}_{s}(Z), S \supset S_{Z}^{\perp}$. Since $S_{0}$ is a maximal $H_{0}$-totally isotropic $F_{0}$-subspace of $S$, we have $S \supset S_{0} \supset S_{Z}^{\perp}$, and $S_{0}$ is its own $H_{0}$-orthogonal complement in $Z$. Thus, if $\sigma=\sigma(\mu, t, b) \in A$, then $t \in S_{0}$. In particular, $\mu$ fixes $S \supset S_{Z}^{\perp}$ and $t \in S$, so that $\sigma$ fixes $X=\langle x\rangle \oplus S_{Z}^{\perp}$, a totally isotropic subspace of dimension $\frac{1}{2}(n-1)$, and also fixes $X^{\perp} / X$. Choose $z \in S_{0}$, $z \notin S_{Z}^{\perp}$. Since $w \rightarrow H(w, z)$ is a nonzero linear map of $S$ on $F$, there exists $w \in S$ such that $H(w, z)=1$. Since the image of $z$ spans the $F_{0}$-space $S_{0} / S_{Z}^{\perp}$, we see that $H(w, t) \in F_{0}$, for all $t \in S_{0}$. Let $\mathcal{L}$ be the $F_{0}$-space of linear transformations of $X^{\perp} / X$ into $X$ spanned by the transformation $\tau$ such that $\tau(\bar{w})=x$, where $\bar{w}$ is the image of $w$ in $X^{\perp} / X$. If $\sigma=\sigma(\mu, t, b) \in A$, then

$$
(\sigma-1) z=-H(z, t) x, \quad z \in S,
$$

so that the linear transformation of $X^{\perp} / X$ into $X$ induced by $\sigma-1$ is equal to

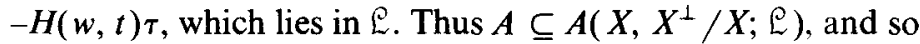

$$
A=A\left(X, X^{\perp} / X ; \mathfrak{Q}\right) \text {. }
$$

Finally, by the proof of Theorem 1, we have the case $s=\frac{1}{2}(n-3), n \geqslant 5$. Then $S$ is totally isotropic, $S_{0}=S$. Since $A_{1} \in \mathbb{Q}\left(Z, \frac{1}{2}(n-3)\right)$ and $f\left(n-2, \frac{1}{2}(n-1)\right)$ $<f\left(n-2, \frac{1}{2}(n-3)\right), S$ is the subspace of all vectors of $Z$ fixed by $A_{1}$. By inductive hypothesis, $A_{1}$ consists of all $\mu \in U(Z)$ which fix $S$ and $S_{Z}^{\perp} / S$, for which the linear transformation of $S_{\bar{Z}} \frac{1}{S} S$ induced by $\mu-1$ lies in a fixed $F_{0}$-space $\mathcal{L}_{1}$ of linear transformations of $F_{0}$-dimension 1 . Let $w \in S_{Z}^{\perp}, w \notin S, 0 \neq \tau \in \mathscr{Q}_{1}$, $u=\tau(\bar{w})$, where $\bar{w}$ is the image of $w$ in $S_{Z}^{\perp} / S$. If $\mu \in A_{1}$, then $(\mu-1) w=c(\mu) u$, where $c(\mu) \in F_{0}$. If $\sigma=\sigma(\mu, t, b) \in A$, then $\mu \in A_{1}$ and $t \in S_{Z}^{\perp}$, since $S_{Z}^{\perp}$ is the $H_{0}$-orthogonal complement of $S_{0}$. Hence $\sigma$ fixes $X=\langle x\rangle \oplus S$ and also $X^{\perp} / X$. Also,

$$
(\sigma-1) w=(\mu-1) w-H(\mu w, t) x=c(\mu) u-H(w, t) x,
$$

since $\mu$ fixes $S_{Z}^{\perp} / S$ and $t \in S_{Z}^{\perp}$.

As remarked before, every element of $\mathcal{L}_{1}$ is induced from some element of $A_{1}$. Hence there exists $\mu^{\prime} \in A_{1}$ such that $c\left(\mu^{\prime}\right)=1$. There exist $t^{\prime}, b^{\prime}$ such that $\sigma^{\prime}=\sigma\left(\mu^{\prime}, t^{\prime}, b^{\prime}\right) \in A$. We have $t \equiv a w(\bmod S), t^{\prime} \equiv a^{\prime} w(\bmod S)$, for some $a, a^{\prime}$ $\in F$. Then, $(\mu-1) t^{\prime}=(\mu-1) a^{\prime} w=a^{\prime} c(\mu) u,\left(\mu^{\prime}-1\right) t=a c\left(\mu^{\prime}\right) u=a u$. Hence $a^{\prime} c(\mu) u=a u$, by Lemma 4 , so that $a=a^{\prime} c(\mu)$. Now $H(w, t)=H(w, a w)=$ $a^{J} H(w, w)=c(\mu) k$, where $k=\left(a^{\prime}\right)^{J} H(w, w)$. Thus, $(\sigma-1) w=c(\mu)(u-k x)$, and we see that $A \subseteq A\left(X, X^{\perp} / X ; \varrho\right)$, where $巳$ is the $F_{0}$-space of linear transformations of $X^{\perp} / X$ into $X$ spanned by the map that takes $\bar{w}$ to $u-k x$. Hence $A=A\left(X, X^{\perp} / X ; \mathfrak{L}\right)$. This completes the proof of Theorem 2 . 
All the groups occurring in Theorem 2 are elementary abelian, except that, in odd dimension and characteristic 2 , the groups $A\left(X, X^{\perp} / X ; \mathcal{L}\right)$ have exponent 4 . In this case, it can be shown that the elementary abelian 2-subgroups of largest order in $U(V)$ are the groups in $\mathcal{Q}\left(V, \frac{1}{2}(n+1)\right)$.

\section{Sylow groups}

As in Wong (1981), a Sylow $p$-subgroup $P$ of $U(V)$ can be described in terms of a sequence of totally isotropic subspaces of $V$,

$$
0=W_{0} \subset W_{1} \subset W_{2} \subset \cdots \subset W_{m},
$$

such that $\operatorname{dim} W_{i}=i$, where $m=\left[\frac{1}{2} n\right]$. Then $P$ consists of those elements of $U(V)$ which fix all $W_{i} / W_{i-1}(1 \leqslant i \leqslant m)$ and $W_{m}^{\perp} / W_{m}$ (in case $n$ is odd), and we write

$$
P=P\left(W_{0}, W_{1}, \ldots, W_{m}\right) \text {. }
$$

We now determine the abelian subgroups of largest order in $P$.

LEMMA 9. Let $X$ be a totally isotropic subspace in $V$, and $Y$ a subspace of $V$ which is invariant under $A\left(X^{\perp}\right)$. Then either $Y \supseteq X$ or $Y \subseteq X^{\perp}$.

Proof. Suppose $Y \nsubseteq X^{\perp}$, and let $y \in Y, y \notin X$. Let $x \in X$. There exists a nonzero element $a$ of $F$ such that $a H(x, y) \in F_{0}$. Then

$$
H(y, y)=H(a x+y, a x+y),
$$

and so there is an isometry of $X^{\perp} \oplus\langle y\rangle$ on $X^{\perp} \oplus\langle a x+y\rangle$, which is the identity on $X^{\perp}$ and maps $y$ on $a x+y$. By Witt's theorem (Dieudonné (1955), page 21), this map extends to an element of $A\left(X^{\perp}\right)$. Since $y \in Y$ and $Y$ is invariant under $A\left(X^{\perp}\right)$, we see that $a x+y \in Y$, and so $x \in Y$. Thus, $X \subseteq Y$. This proves Lemma 9.

THEOREM 3. Let $P=P\left(W_{0}, W_{1}, \ldots, W_{m}\right)$ be a Sylow p-subgroup of the unitary group $U(V)$ of a vector space $V$ of dimension $n=2 m$ or $2 m+1(m \geqslant 1)$ over $a$ finite field $F$ of $q^{2}$ elements having characteristic $p$. Let $Q(P)$ be the set of all abelian subgroups of largest order in $P$, and let $F_{0}$ be the subfield of $q$ elements in $F$.

(a) If $n=2 m$, then $\mathbb{Q}(P)$ consists of the single group $A\left(W_{m}\right)$.

(b) If $n=2 m+1$, then $Q(P)$ consists of all groups $A\left(W_{m}, W_{m}^{\perp} / W_{m} ; \mathcal{L}\right)$, where $\mathcal{L}$ is an $F_{0}$-subspace of $F_{0}$-dimension 1 in the space of all linear maps of $W_{m}^{\perp} / W_{m}$ into $W_{m}$. There are $\left(q^{2 m}-1\right) /(q-1)$ such groups. 
Proof. Suppose $A \in \mathscr{Q}(P)$. By Theorem 2, $A$ has the form

$$
A(X) \text { or } A\left(X, X^{\perp} / X ; \varrho\right) \text {, }
$$

where $X$ is totally isotropic of dimension $m$. In any case, $A \supseteq A\left(X^{\perp}\right)$, and so $W_{m}$ is invariant under $A\left(X^{\perp}\right)$. If $W_{m} \subseteq X^{\perp}$, then $X+W_{m}$ is totally isotropic, and so $X \subseteq W_{m}$, since $W_{m}$ is a maximal totally isotropic subspace of $V$. By Lemma 9 , we have $X \subseteq W_{m}$ in any case, and so $X=W_{m}$.

Conversely, every group $A\left(W_{m}\right)$ or $A\left(W_{m}, W_{m}^{\perp} / W_{m}\right.$; $\left.巳\right)$ clearly lies in $P\left(W_{0}, W_{1}, \ldots, W_{m}\right)$.

In case (b), the vector space of all linear maps of $W_{m}^{\perp} / W_{m}$ into $W_{m}$ has dimension $m$ over $F$, and so there are $\left(q^{2 m}-1\right) /(q-1)$ possible choices for the $F_{0}$-subspace $\mathcal{L}$ of $F_{0}$-dimension 1 . This proves Theorem 3 .

The determination of $Q(P)$ for the unitary case has also been carried out in the Ph.D. thesis of Michael Barry (1977), using the Chevalley commutator relations.

It is easy to check which of the groups in $Q(P)$ are normal in $P$. We give the result without proof.

THEOREM 4. In Theorem 3, let $\mathbb{Q}_{N}(P)$ denote the set of groups in $Q(P)$ which are normal in $P$.

(a) If $n$ is even, then $Q_{N}(P)=\mathscr{Q}(P)$.

(b) If $n$ is odd and $n \geqslant 3$, then $\mathbb{Q}_{N}(P)$ consists of the $q+1$ groups of the form $A\left(W_{m}, W_{m}^{\perp} / W_{m} ; \mathfrak{L}\right)$, where $\mathcal{L}$ is an $F_{0}$-subspace of $F_{0}$-dimension 1 in the space of all linear transformations of $W_{m}^{\perp} / W_{m}$ into $W_{1}$.

\section{Symplectic case, $p \neq 2$}

Now suppose $J=1, p \neq 2$, so that $H$ is an alternating form, and let $|F|=q$. Assume $V$ is nondegenerate so that $V$ has even dimension $2 m$, and let $q^{f(m, r)}$ denote the largest order of an abelian $p$-subgroup of $\mathrm{Sp}(V)$ fixing an $r$-dimensional subspace of $V$.

We give only a sketch of part of the argument in this case, since the method is just a simpler version of that used for the unitary case. Lemmas 3 and 4 hold for $\operatorname{Sp}(V)$ in place of $U(V)$, where now $B(t)=F$ for all $t$. If $A$ is an abelian $p$-subgroup of $\operatorname{Sp}(V)$ fixing an $r$-dimensional subspace $X$, and $x$ is a nonzero vector in $\operatorname{rad} X$, then $S$ can be defined as in Lemma 5 , and $s=\operatorname{dim} S$ satisfies $0 \leqslant s \leqslant 2 m-r-1$. The analogue of $H_{0}$ is $2 H$, which is nondegenerate because 
$p \neq 2$, and we see that $S$ is totally isotropic, so that $s \leqslant m-1$. The analogue of Lemma 8 , for $1 \leqslant r<2 m$, is that

$$
f(m, r)=\max \{g(m, r, s) \mid 0 \leqslant s \leqslant \min (2 m-r-1, m-1)\},
$$

where $g(m, r, s)=1+s+f(m-1, \max (r-1, s))$. Induction on $m$ leads to the following result.

THEOREM 5. Let $\mathrm{Sp}(V)$ be the symplectic group of a $2 m$-dimensional vector space $V$ over a field $F$ of $q$ elements having odd characteristic $p$, and let $q^{f(m, r)}$ be the largest order of an abelian p-subgroup of $\mathrm{Sp}(V)$ fixing an r-dimensional subspace of V.

(a) If $r \geqslant m$, then $f(m, r)=\frac{1}{2}(2 m-r)(2 m-r+1)$.

(b) If $r<m$, then $f(m, r)=\frac{1}{2} m(m+1)$.

As before, we denote by $\bar{s}_{r}(V)$ the set of all $r$-dimensional subspaces $X$ of $V$ such that $X \subseteq X^{\perp}$ or $X \supseteq X^{\perp}$, and we write $A(X)$ for the subgroup of $\operatorname{Sp}(V)$ of all elements fixing $X$. The following result is proved in the same way as Theorem 2(a), (b).

THEOREM 6. Let $\mathrm{Sp}(V)$ be the symplectic group of a 2 m-dimensional vector space $V$ over a finite field of odd characteristic $p$, and let $\mathbb{Q}(V, r)$ be the set of all abelian p-subgroups of $\mathrm{Sp}(V)$ of largest order fixing an $r$-dimensional subspace of $V$.

(a) If $r \geqslant m$, then $Q(V, r)=\left\{A(X) \mid X \in \S_{r}(V)\right\}$.

(b) If $r<m$, then $\mathbb{Q}(V, r)=\mathbb{Q}(V, m)$.

All the groups occuring in Theorem 6 are elementary abelian.

A Sylow $p$-subgroup $P$ of $\mathrm{Sp}(V)$ is determined by a sequence of totally isotropic subspaces

$$
0=W_{0} \subset W_{1} \subset W_{2} \subset \cdots \subset W_{m},
$$

such that $\operatorname{dim} W_{i}=i ; P$ is the group $P\left(W_{0}, W_{1}, \ldots, W_{m}\right)$ of all elements of $\operatorname{Sp}(V)$ fixing all $W_{i} / W_{1-1}(1 \leqslant i \leqslant m)$. The following result is proved in the same way as Theorem 3(a).

TheOREM 7. Let $P=P\left(W_{0}, W_{1}, \ldots, W_{m}\right)$ be a Sylow p-subgroup of the symplectic group $\operatorname{Sp}(V)$ of a $2 m$-dimensional vector space $V$ over a finite field of odd characteristic $p$. Then, $A\left(W_{m}\right)$ is the unique abelian subgroup of largest order in $P$.

The last result was also proved by Barry (1979) by using the Chevalley commutator relations. 


\section{References}

M. J. J. Barry (1977), 'Parabolic subgroups of groups of Lie type', Ph.D. thesis, University of Notre Dame.

M. J. J. Barry (1979), 'Large abelian subgroups of Chevalley groups'. J. Austral. Math. Soc. Ser. A 27, $59-87$.

M. J. J. Barry and W. J. Wong (1982), 'Abelian 2-subgroups of finite symplectic groups in characteristic 2', J. Austral. Math. Soc. Ser. A.

J. Dieudonné (1955), La géométrie des groupes classiques (Springer-Verlag, Berlin).

J. T. Goozeff (1970), 'Abelian $p$-subgroups of the general linear group', J. Austral. Math. Soc. 11, 257-259.

W. J. Wong (1981), 'Abelian unipotent subgroups of finite orthogonal groups', J. A ustral. Math. Soc. Ser. A 32, 223-245.

Department of Mathematics

University of Notre Dame

Notre Dame, Indiana 46556

U.S.A. 